
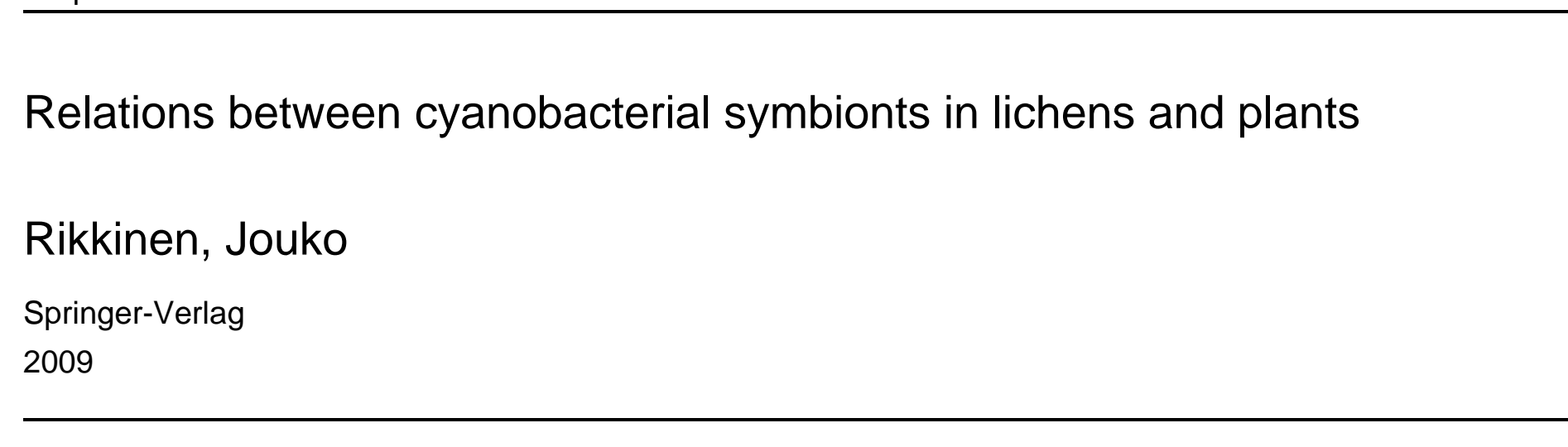


\title{
Relations Between Cyanobacterial Symbionts in Lichens and Plants
}

Jouko Rikkinen

Department of Biological and Environmental Sciences, University of Helsinki, 65, 00014 Helsinki, Finland

\begin{abstract}
Cyanobacteria participate in many types of symbioses, either serving as a source of fixed carbon and nitrogen, as in many cyanolichens, or solely as a source of nitrogen, as in plant symbioses. Symbiotic strains of Nostoc are by far the most common cyanobionts in lichens and related strains are also found in thalloid bryophytes, cycads, and in the angiosperm Gunnera. This article provides a short summary of Nostoc diversity patterns in lichens and argues that symbiont-switches between lichen-forming fungi and plants may have played a role in the evolution of some extant cyanobacterial symbioses.
\end{abstract}

\section{Introduction}

Lichen-forming species represent a major ecological group among the Ascomycota, with over 13 500 lichenized species. While a great majority of these fungi associate with green algae, over 1500 species of lichen-forming fungi have cyanobacteria as primary or accessory photobionts. The

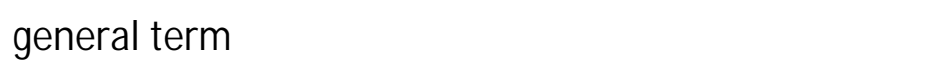

the sole photosymbiotic component, or as an accessory photobiont in addition to green algae.

Cyanolichens are often divided into two artificial groups: the bipartite and tripartite species. Bipartite cyanolichens are stable symbioses between a single species of lichen-forming fungus and one cyanobacterial photobiont. In most of these lichens the cyanobiont forms a more or less continuous layer immediately below the upper cortex of the lichen thallus (Fig. 1). Tripartite cyanolichens, on the other hand, house both green algal and cyanobacterial symbionts. In these lichens the cyanobionts, which usually represent a minor proportion of photobiont biomass, are restricted to special structures called cephalodia (Fig. 1). Over 500 lichen species are known to produce internal or external cephalodia. In addition, many green algal lichens associate with Yå 0] dVP_«V\} fixed nitrogen. Sometimes the free-living cyanobacteria are totally covered by fungal hyphae, and the resulting compound structures are called paracephalodia (Poelt and Mayhofer 1988). 
The mycobionts of certain cephalodiate lichens may sometimes produce different thallus morphotypes in symbiosis with compatible green algae and cyanobacteria. Chimeroid lichens with green algae and cyanobacteria as primary photobionts in different parts of the same thallus are called photosymbiodemes (Fig. 1). The contrasting morphotypes may either combine into a compound thallus or, in some cases, live separate lives. Photosymbiodemes offer unique opportunities to compare the physiological performances of green algal and cyanobacterial lichen photobionts under almost identical conditions of habitat, growth history, and fungal association (Demmig-Adams et al. 1990; Green et al. 1993, 2002). On the whole, however, photosymbiodemes are rare and only occur in some species of Lobaria, Nephroma, Peltigera, Pseudocyphellaria, and Sticta.

Lichen-symbiotic cyanobacteria have the potential to give photosynthate and/or fixed nitrogen to their fungal partners (Palmqvist 2002; Rai 2002). The relative importance of these activities is known to vary between bi- and tripartite lichens. The cyanobionts of bipartite lichens usually show lower heterocyst frequencies and lower rates of nitrogen fixation than those of tripartite species. In tripartite cyanolichens, the cyanobiont often shows a high rate of nitrogen fixation, while the green algal photobiont typically produces most of the photosynthate. Both bipartite and tripartite lichen symbioses have clearly evolved repeatedly in different lineages of fungi and convergent evolution has often led to similar thallus structures in distantly related cyanolichens (Rikkinen 2002).

\section{Lichen-forming and Plant-associated Nostoc Strains}

Serious attempts to determine the strain identity of the cyanobacterial photobiont have only been made for a small fraction of all cyanolichen species. However, different strains of Nostoc are known to occur in many cyanolichens, especially in association with lichen-forming species of the Lecanorales (Ascomycota). Typical strains of Nostoc produce isopolar trichomes with no evidence of branching or meristematic zones, and their cells are cylindrical or spherical. Also the characteristic life-cycle, with motile hormogonia and vegetative filaments is shown by most strains in culture. However, some symbiotic strains do not produce hormogonia under common growth conditions.

Recent studies in Europe (Paulsrud and Lindblad 1998; Paulsrud et al. 1998, 2001; Oksanen et al. 2002, 2004; Lohtander et al. 2003), North America (Paulsrud et al. 200ї K [ [qüX D̃ U 
East Asia (Rikkinen et al. 2002), New Zealand (Summerfield et al. 2002), South-America (Stenroos et al. 2006), and Antarctica (Wirtz et al. 2003) have shown that cyanolichens house a wide variety of symbiotic Nostoc genotypes. Further diversity has been found from hornwort, liverwort and cycad symbioses (Costa et al. 1999, 2001), and from macroscopic colonies of non-symbiotic Nostoc (Wright et al. 2001).

All evidence indicates that the Nostoc symbionts of cyanolichens are closely related to plantsymbiotic and free-living strains. Together these or ganisms form a genetically diverse, but rather well-delimited, monophyletic group among the Nostocalean cyanobacteria. Most strains of Nostoc s.str. can be conveniently assigned to two broad groups. The first group is genetically heterogeneous and includes the cyanobionts of many predominately terricolous cyanolichens, including those of all tripartite Nephroma and Peltigera species, but also the Nostoc symbionts of thalloid bryophytes and cycads, many free-living strains, etc. The second group is genetically less diverse and seems to only contain cyanobionts of bipartite, predominately epiphytic or lithophytic cyanolichensv their fungal hosts, however, are variable and represent many different groups among the Ascomycota.

Studies have shown that lichen-forming fungi are highly selective with respect to their cyanobionts. Only a few closely related Nostoc strains typically serve as the appropriate symbiotic partners for each fungal species. On the other hand, many different fungi, often from different genera or even families, can exploit and potentially share specific cyanobacterial strains. Thus, some cyanolichens may form photobiont-mediated guilds, i.e. co-occurring populations of lichenforming fungi that utilize a common symbiont. Some lichen-forming fungimay even depend on other guild members for the effective dispersal of their cyanobionts (Rikkinen et al. 2002). While the ecological boundaries between existing guilds may appear steep, on an evolutionary timescale they are easily crossed. For a lichen-forming fungus, each successful shift into a new guild has provided new opportunities for specialization and subsequent radiation. Furthermore, each shifting fungus has had the potential to change pre-existing ecological balances between previous guild members (Rikkinen 2003).

\section{Concluding Remarks}

In the present context it is particularly significant that during evolution many lichen-forming fungi may have formed novel associations with plantsymbiotic Nostoc strains and/or vice versa. One 


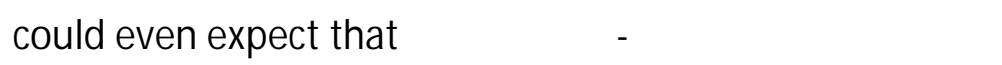

lichens, thalloid bryophytes, and/or cycads. The cyanobionts of most terricolous lichens belong to a specific group of symbiotic Nostoc strains. Related cyanobionts are also found in the coralloid roots of cycads, in the symbiotic slime cavities of hornworts, and in the symbiotic auricles of thalloid liverworts. The two thalloid liverworts with Nostoc symbionts, Blasia pusilla and Cavicularia densa, occupy a very basal position among the complex thalloid liverworts. These plants, the hornworts and the cycads together represent a conspicuous proportion of all extant plant lineages that can be reliably traced back to pre-Permian times. Concurrently, among the Fungi, some terricolous lichens are believed to be of ancient origin. Considering all this antiquity, it may not be a coincidence that all the hosts rely on related cyanobacterial symbionts.

\section{References}

Costa JL, Paulsrud P, Lindblad P (1999) Cyanobiont diversity within coralloid roots of selected cycad species. FEBS Microbiol Ecol 28:85t 91

Costa J L, Paulsrud P, Rikkinen J, Lindblad P (2001) Genetic diversity of Nostoc endophytically associated with two bryophyte species. Appl Environ Microbiol 67:4393t 4396

Demmig-Adams B, Adams WW III, Green TGA, Czygan FC, Lange OL (1990) Differences in the susceptibility to light stress in two lichens forming a phycosymbiodeme, one partner possessing and one lacking the xanthophyll cycle. Oecologia 84:451t 456

Green TGA, Büdel B, Heber U, Meyer A, Zellner H, Lange OL (1993) Differences in photosynthetic performance between cyanobacterial and green algal components of lichen 

